\title{
Young integrals and SPDEs
}

\author{
Antoine Lejay \\ Project OMEGA, INRIA Lorraine \\ IECN, Campus Scientifique \\ BP 239 - 54506 Vandoeuvre-lès-Nancy CEDEX, France \\ Antoine.Lejay@iecn.u-nancy.fr \\ Massimiliano Gubinelli \\ Laboratoire Analyse, Géométrie \&3 Applications - UMR 7539 \\ Institute Galilée, Universitè Paris 13, \\ 93430 - Villetaneuse, France \\ gubinell@math. univ-paris13.fr \\ Samy Tindel \\ Institut Elie Cartan \\ Université Henri Poincaré (Nancy) \\ BP 239 - 54506 Vandouvre-lès-Nancy CEDEX, France \\ tindel@iecn.u-nancy.fr
}

October 24, 2018

\begin{abstract}
In this note, we study the non-linear evolution problem

$$
d Y_{t}=-A Y_{t} d t+B\left(Y_{t}\right) d X_{t}
$$

where $X$ is a $\gamma$-Hölder continuous function of the time parameter, with values in a distribution space, and $-A$ the generator of an analytical semigroup. Then, we will give some sharp conditions on $X$ in order to solve the above equation in a function space, first in the linear case (for any value of $\gamma$ in $(0,1)$ ), and then when $B$ satisfies some Lipschitz type conditions (for $\gamma>1 / 2$ ). The solution of the evolution problem will be understood in the mild sense, and the integrals involved in that definition will be of Young type.
\end{abstract}




\section{Introduction}

The existence and uniqueness problem for ordinary differential equations driven by an irregular path of Hölder continuity greater than $1 / 2$ is now fairly well understood, either in the context of fractional integrals (Zäh98), or as a first step towards the definition of differential equations driven by a rough path (see (Gub04; Lei03; LQ02)).

However, the case of partial differential equations of evolution type has only been partially treated. For instance, when the driving noise is an infinite dimensional fractional Brownian motion of Hurst parameter $H$, let us mention two results that have been obtained recently:

- In case of a linear equation with additive noise, some optimal conditions on the space covariance of the noise, ensuring the existence and uniqueness of a function-valued solution to the evolution equation, are given in (TTV03). These results are based on a representation of the solution involving some Skorokhod type integrals, that cannot be interpreted as the limit of Riemann sums.

- In the non-linear case, the same kind of results are available in (MN03). In this latter case, only the case $H>1 / 2$ is considered, the space covariance is assumed to be trace class (which implies a strong regularity condition on the driving noise) and the integrals are defined path-wise.

On the other hand, some differential equations in a Banach space, driven by a rough path, are considered in (LLQ02), but the question of the regularization of the noise by a semi-group is not addressed in that last reference.

With all those facts in mind, our aim, in this paper, is to make a step towards the resolution of non linear partial differential equations driven by an infinite dimensional rough path. To be more specific, let us start by defining roughly the kind of equation we handle: we consider an unbounded operator $A$ on a Banach space $\mathcal{B}$, and assume that $-A$ is the infinitesimal generator of an analytical semi-group $(S(t))_{t \geq 0}$. This induces a family of Banach spaces $\left\{\mathcal{B}_{\alpha} ; \alpha \in \mathbb{R}\right\}$, where, for $\alpha>0, \mathcal{B}_{\alpha}=\operatorname{Dom}\left(A^{\alpha}\right)$, and $\mathcal{B}_{-\alpha}$ is defined by duality. Now, our noise $X$ is a function from $[0, T]$ to $\mathcal{B}_{-\alpha}$, for a certain $\alpha>0$, with a given Hölder continuity $\gamma \in(0,1)$ in time. Our equation is of the form

$$
\mathrm{d} Y_{t}=-A Y_{t} \mathrm{~d} t+B\left(Y_{t}\right) \mathrm{d} X_{t}, \quad t \in[0, T],
$$

with a given initial condition $y_{0}$, and where $B$ is a map from $\mathcal{B}_{\delta}$ to $\mathcal{L}\left(\mathcal{B}_{-\alpha} ; \mathcal{B}_{\delta}\right)$ satisfying some (local) Lipschitz conditions. In fact, equation (11) is understood in the so-called mild sense, i.e. we say that $Y$ is a solution to (11) in 
$[0, T]$ if it is a function in $C^{\kappa}\left([0, T] ; \mathcal{B}_{\delta}\right)$, with a given $\kappa>0$, satisfying

$$
Y(t)=S(t) y_{0}+\int_{0}^{t} S(t-s) B(Y(s)) \mathrm{d} X(s), \quad t \leq T .
$$

Based on these notations, we get the following results:

1. In the linear additive case, that is when $B=\mathrm{Id}$, we get the existence and uniqueness of a global solution to (1), living in $C^{\kappa}\left([0, T] ; \mathcal{B}_{\delta}\right)$ for any $T>0$, with the following condition on the coefficients $\alpha, \delta, \gamma$ and $\kappa$ :

$$
\alpha<\gamma, \quad 0<\delta<\gamma-\alpha, \quad 0<\kappa<\gamma-\alpha-\delta .
$$

This result is given in a rigorous form at Theorem 1

2. In the general case, if $B$ satisfies some Lipschitz type conditions, and under the additional assumption $\gamma+\delta>1$ (which implies in particular that $\gamma>1 / 2$ ), we also get the existence and uniqueness of the solution to (11). In case of a locally Lipschitz coefficient $B$, the existence of a solution can only be guaranteed up to an explosion time $T>0$.

3. Eventually, we check that our abstract results can be applied to a simple case, namely the case of the heat equation in $[0,1]$ with Dirichlet boundary conditions. We also assume that $X$ is a cylindrical fractional Brownian noise (see Section 4.1 for a complete description). Then we are able to solve the equation (133) for a function $\sigma \in C_{b}^{2}(\mathbb{R})$, up to a strictly positive explosion time. At that point, let us insist again on the following fact: we are allowed to consider, in the case of equation (33), a white noise in space for any $H>1 / 2$. To our knowledge, this is the first occurrence of an existence and uniqueness result for a non linear SPDE driven by a fractional Brownian motion with a non trivial space covariance.

Let us also observe that, in order to get those results, we use a quite natural approach: our setting allows us to use Young integrals, that can be approximated by Riemann sums. Then, in each of those Riemann sums, we regularize the path $X_{t} \in \mathcal{B}_{-\alpha}$ by the semi-group $S$ in order to get an element of $\mathcal{B}_{\delta}$. This induces an additional singularity in time, that we compensate by the Hölder regularity of $X$. Of course, one may want to consider some more irregular paths, in which case one could expect to need paths living in a "bigger" space that $\mathcal{B}_{-\alpha}$, as in the rough path theory. But we believe that the results contained in this article may represent one of the corner stone for the development of such a general theory. 
Our paper is organized as follows: at Section 2, we recall some basic facts about analytic semi-groups, and treat the linear case. Section 3 is devoted to the non-linear case, and Section 4 to the example of the heat equation in dimension 1.

\section{Case of an additive noise}

In this Section, we first recall some basic facts about analytical semi-groups, that we will use throughout the paper, and then solve equation (11) in the linear additive case.

\subsection{Analytical semi-group}

This section contains some classical results about analytical semi-groups and fractional power of their infinitesimal generators. For proofs, see (Paz83; EN00; Fat99) for example.

Let $(\mathcal{B},\|\cdot\|)$ be a separable Banach space. Let $(A, \operatorname{Dom}(A))$ be a nonbounded linear operator on $\mathcal{B}$ such that $-A$ be the infinitesimal generator of an analytical semi-group $(S(t))_{t>0}$. Assume that for some constants $M>0$ and $\lambda<0,\|S(t)\|_{\mathcal{B} \rightarrow \mathcal{B}} \leq M e^{-\lambda t}$ for all $t \geq 0$. In particular, this implies that $A$ is one-to-one from $\operatorname{Dom}(A)$ to $\mathcal{B}$.

For any $\alpha \in \mathbb{R}$, the fractional power $\left(A^{\alpha}, \operatorname{Dom}\left(A^{\alpha}\right)\right)$ of $A$ can be defined. If $\alpha<0$, then $\operatorname{Dom}\left(A^{\alpha}\right)=\mathcal{B}$ and $A^{\alpha}$ is one-to-one from $\mathcal{B}$ to $\operatorname{rg}\left(A^{\alpha}\right)$ and is a bounded operator. Besides, if $\alpha \geq 0,\left(A^{\alpha}, \operatorname{Dom}\left(A^{\alpha}\right)\right)$ is a closed operator with a dense domain $\operatorname{Dom}\left(A^{\alpha}\right)=\operatorname{rg}\left(A^{-\alpha}\right)$. Moreover, $A^{\alpha}=\left(A^{-\alpha}\right)^{-1}$.

For $\alpha \geq 0$, let $\mathcal{B}_{\alpha}$ be the space $\operatorname{Dom}\left(A^{\alpha}\right)$ with the norm $\|x\|_{\mathcal{B}_{\alpha}}=\left\|A^{\alpha} x\right\|$. Since $A^{-\alpha}$ is continuous, it follows that the norm $\|\cdot\|_{\mathcal{B}_{\alpha}}$ is equivalent to the graph norm of $A^{\alpha}$. If $\alpha=0$, then $\mathcal{B}_{\alpha}=\mathcal{B}$ and $A^{0}=$ Id.

If $\alpha<0$, let $\mathcal{B}_{\alpha}$ be the completion of $\mathcal{B}$ with respect to $\|x\|_{\mathcal{B}_{\alpha}}=\left\|A^{\alpha} x\right\|$. Thus, $\mathcal{B}_{\alpha}$ is a larger space than $\mathcal{B}$.

Among the important facts about these spaces, note the following ones: For any $\alpha \in \mathbb{R}$ and any $\rho \geq 0$,

$$
\begin{gathered}
A^{-\rho} \text { maps } \mathcal{B}_{\alpha} \text { onto } \mathcal{B}_{\alpha+\rho}, \text { for all } \alpha \in \mathbb{R}, \rho \geq 0, \\
\|x\|_{\mathcal{B}_{\alpha}} \leq C_{\alpha, \delta}\|x\|_{\mathcal{B}_{\delta}} \text { for all } x \in \mathcal{B}_{\alpha} \text { and all } \alpha \leq \delta .
\end{gathered}
$$

Moreover, for all $\alpha, \beta \in \mathbb{R}$,

$$
A^{\alpha} A^{\beta}=A^{\alpha+\beta} \text { on } \mathcal{B}_{\gamma}
$$

with $\gamma=\max \{\alpha, \beta, \alpha+\beta\}$. 
The semi-group $(S(t))_{t \geq 0}$ also satisfies

$$
\begin{gathered}
S(t) \text { may be extended to } \mathcal{B}_{\alpha} \text { for all } \alpha<0 \text { and all } t \geq 0, \\
\qquad(t) \text { maps } \mathcal{B}_{\alpha} \text { to } \mathcal{B}_{\delta} \text { for all } \alpha \in \mathbb{R}, \delta \geq 0, \\
\text { for all } t>0, \alpha \in \mathbb{R},\left\|A^{\alpha} S(t)\right\|_{\mathcal{B} \rightarrow \mathcal{B}} \leq M_{\alpha} t^{-\alpha} e^{-\lambda t} \\
\text { for } 0<\alpha \leq 1, x \in \mathcal{B}_{\alpha},\|S(t) x-x\| \leq C_{\alpha} t^{\alpha}\left\|A^{\alpha} x\right\|
\end{gathered}
$$

where with $\|\cdot\|_{\mathcal{B} \rightarrow \mathcal{B}}$ we denote the operator norm from $\mathcal{B}$ to $\mathcal{B}$.

\subsection{Mild solutions of the Cauchy problem with an ad- ditive noise}

This subsection is devoted to the linear additive case of our evolution equation, for which we will introduce first some additional notation: for any function $f$ defined on $\mathbb{R}_{+}$, set $f(s, t)=f(t)-f(s)$. For a $\gamma$-Hölder continuous function $f$ from $[0, T]$ to a Banach space $\mathcal{X}$, define $H_{\gamma, T}(f ; \mathcal{X})$ by

$$
H_{\gamma, T}(f ; \mathcal{X})=\sup _{0 \leq s \leq t \leq T} \frac{\|f(s, t)\|_{\mathcal{X}}}{(t-s)^{\gamma}} .
$$

Let $Y$ be a $\gamma$-Hölder continuous function from $[0, T]$ to $\mathcal{B}$ with $\gamma \in(0,1)$, and set $H(Y)=H_{\gamma, T}(Y ; \mathcal{B})$. Fix also $\alpha \in \mathbb{R}$ and set $X=A^{\alpha} Y$. Note that $X$ belongs to $\mathcal{B}_{-\alpha}$, but does not necessarily belong to $\mathcal{B}$.

Consider now the following Cauchy linear equation

$$
u(t)=X(t)-\int_{0}^{t} A u(s) \mathrm{d} s .
$$

Without loss of generality, it can be assumed that $X(0)=0$. Otherwise, the solution $u$ to (91) is the sum of $v$ and $w$ that are solutions to $v(t)=$ $X(t)-X(0)-\int_{0}^{t} A v(s) \mathrm{d} s$ and $w(t)=X(0)-\int_{0}^{t} A w(s) \mathrm{d} s$. The solution of the later problem is $w(t)=S(t) X(0)$. If $X$ is smooth enough and $X^{\prime}$ belongs to $\mathrm{L}^{1}([0, T] ; \mathcal{B})$, then

$$
v(t)=\int_{0}^{t} S(t-s) X^{\prime}(s) \mathrm{d} s
$$

belongs to $\mathcal{C}([0, T] ; \mathcal{B})$ and is called a mild solution of (9). This notion of solution is weaker than the notion of strong solutions, since $v$ is not necessarily differentiable. On the relation between strong and mild solutions, see for example (Paz83).

We can now state the main result of this section: 
Theorem 1. Assume that $\alpha<\gamma$. There exists a linear map

$$
\mathfrak{S}: \mathcal{C}^{\gamma}\left([0, T] ; \mathcal{B}_{-\alpha}\right) \rightarrow \mathcal{C}^{\kappa}\left([0, T] ; \mathcal{B}_{\delta}\right)
$$

for all $\delta \in(0, \gamma-\alpha)$ and all $\kappa \in(0,(\gamma-\alpha-\delta) \wedge 1)$ such that, if $X$ belongs to $\mathcal{C}^{1}\left([0, T] ; \mathcal{B}_{-\alpha}\right)$,

$$
\mathfrak{S}(X)(t)=\int_{0}^{t} S(t-s) X^{\prime}(s) \mathrm{d} s .
$$

Moreover, for all $T>0$, there exist some constants $C_{1}$ and $C_{2}$ depending only on $\alpha, \kappa, \delta$ and $\gamma$ such that

$$
\begin{aligned}
& H_{\kappa, T}\left(\mathfrak{S}(X) ; \mathcal{B}_{\delta}\right) \leq C_{1} H_{\gamma, T}\left(X ; \mathcal{B}_{-\alpha}\right) \\
& \text { and } \sup _{t \in[0, T]}\|\mathfrak{S}(X)(t)\|_{\mathcal{B}_{\delta}} \leq C_{2} T^{\gamma-\delta-\alpha} H_{\gamma, T}\left(X ; \mathcal{B}_{-\alpha}\right) .
\end{aligned}
$$

Proof. Fix $T \geq t \geq s^{\prime}>s \geq 0$. Let $n \in \mathbb{N}$ and set

$$
\mathfrak{S}^{n}(X)\left(s, s^{\prime} ; t\right)=\sum_{k=\left\lceil 2^{n} s / t\right\rceil}^{\left\lfloor 2^{n} s^{\prime} / t\right\rfloor-1} S\left(t-t_{k}^{n}\right) X\left(t_{k}^{n}, t_{k+1}^{n}\right),
$$

where $t_{k}^{n}=t k / 2^{n}$ and $X(a, b)=X(b)-X(a)$. Using the semi-group property, the difference between $\mathfrak{S}^{n}(X)(s, t)$ and $\mathfrak{S}^{n}(X)(s, t)$ is

$$
\begin{aligned}
\mathfrak{S}^{n+1}(X) & \left(s, s^{\prime} ; t\right)-\mathfrak{S}^{n}(X)\left(s, s^{\prime} ; t\right) \\
= & \sum_{k=\left\lceil 2^{n} s / t\right\rceil}^{\left\lfloor 2^{n} s^{\prime} / t\right\rfloor-1}\left(S\left(t-t_{2 k+1}^{n+1}\right)-S\left(t-t_{2 k}^{n+1}\right)\right) X\left(t_{2 k+1}^{n+1}, t_{2 k+2}^{n+1}\right) \\
= & \sum_{k=\left\lceil 2^{n} s / t\right\rceil}^{\left\lfloor 2^{n} s^{\prime} / t\right\rfloor-1}\left(\operatorname{Id}-S\left(t_{2 k+1}^{n+1}-t_{2 k}^{n+1}\right)\right) S\left(t-t_{2 k+1}^{n+1}\right) X\left(t_{2 k+1}^{n+1}, t_{2 k+2}^{n+1}\right)
\end{aligned}
$$

Now, fix $\delta<\gamma-\alpha$ and $\beta \in(1-\gamma,(1-\alpha-\delta) \wedge 1)$. With (4) and (7)-(8),

$$
\begin{aligned}
\| A^{\delta} \mathfrak{S}^{n+1}(X)\left(s, s^{\prime} ; t\right) & -A^{\delta} \mathfrak{S}^{n}(X)\left(s, s^{\prime} ; t\right) \| \\
\leq & \sum_{k=\left\lceil 2^{n} s / t\right\rceil}^{\left\lfloor 2^{n} s^{\prime} / t\right\rfloor-1} C_{\beta}\left(\frac{t}{2^{n+1}}\right)^{\beta}\left\|A^{\beta+\delta+\alpha} S\left(t-t_{2 k+1}^{n+1}\right) Y\left(t_{2 k+1}^{n+1}, t_{2 k+2}^{n+1}\right)\right\| \\
\leq & \sum_{k=\left\lceil 2^{n} s / t\right\rceil}^{\left\lfloor 2^{n} s^{\prime} / t\right\rfloor-1} C_{\beta} M_{\alpha+\beta+\gamma}\left(\frac{t}{2^{n+1}}\right)^{\beta+\gamma} \frac{H(Y)}{\left(t-t_{2 k+1}^{n+1}\right)^{\beta+\delta+\alpha}} .
\end{aligned}
$$


Set $\epsilon=\alpha+\delta+\beta$. By assumption, $\epsilon<1$, and furthermore,

$$
\begin{aligned}
\sum_{k=\left\lceil 2^{n} s / t\right\rceil}^{\left\lfloor 2^{n} s^{\prime} / t\right\rfloor-1} \frac{1}{\left(t-t_{2 k+1}^{n+1}\right) \epsilon} & \leq \frac{2^{n+1}}{t^{\epsilon}} \int_{\left\lceil 2^{n} s / t\right\rceil / 2^{n+1}}^{\left\lfloor 2^{n} s^{\prime} / t\right\rfloor / 2^{n+1}} \frac{\mathrm{d} r}{(1-r)^{\epsilon}} \\
& \leq \frac{2^{n+1}}{t^{\epsilon}} \int_{0}^{1} \frac{\mathrm{d} r}{(1-r)^{\epsilon}} .
\end{aligned}
$$

Hence, there exists a constant $C$ that depends only on $\alpha, \beta$ and $\delta$ for which

$$
\left\|A^{\delta} \mathfrak{S}^{n+1}(X)\left(s, s^{\prime} ; t\right)-A^{\delta} \mathfrak{S}^{n}(X)\left(s, s^{\prime} ; t\right)\right\| \leq \frac{C H(Y) t^{\gamma-\delta-\alpha}}{2^{n(\beta+\gamma-1)}}
$$

for all integer $n$. Since $\beta+\gamma>1$, the series

$$
\sum_{n \geq 0}\left\|A^{\delta} \mathfrak{S}^{n+1}(X)\left(s, s^{\prime} ; t\right)-A^{\delta} \mathfrak{S}^{n}(X)\left(s, s^{\prime} ; t\right)\right\|
$$

is convergent, and moreover,

$$
\left\|A^{\delta} \mathfrak{S}^{0}(X)\left(s, s^{\prime} ; t\right)\right\|=\left\|A^{\delta+\alpha} S(t-s) Y\left(s, s^{\prime}\right)\right\| \leq \frac{\left(s^{\prime}-s\right)^{\gamma} H(Y) M_{\alpha+\delta}}{(t-s)^{\delta+\alpha}} .
$$

Thus, the sequence $\left(A^{\delta} \mathfrak{S}^{n}(X)\left(s, s^{\prime} ; t\right)\right)_{n \in \mathbb{N}}$ is convergent in $\mathcal{B}$. Let now $\mathfrak{S}(X)\left(s, s^{\prime} ; t\right)$ be the limit of $\left(\mathfrak{S}^{n}(X)\left(s, s^{\prime} ; t\right)\right)_{n \in \mathbb{N}}$. Observe that, since $A^{\delta}$ is a closed operator, the limit of $\left(A^{\delta} \mathfrak{S}^{n}(X)\left(s, s^{\prime} ; t\right)\right)_{n \in \mathbb{N}}$ is $A^{\delta} \mathfrak{S}(X)\left(s, s^{\prime} ; t\right)$.

To simplify the notations, set $\mathfrak{S}(X)(t)=\mathfrak{S}(X)(0, t ; t)$. The linearity of $X \mapsto \mathfrak{S}(X)(t)$ follows immediately from the construction of $\mathfrak{S}(X)(t)$. The inequality (11) is easily obtained from (12) and (13).

Note also that if $X$ is smooth, then $\mathfrak{S}(X)\left(s, s^{\prime} ; t\right)=\int_{s}^{s^{\prime}} S(t-s) X^{\prime}(s) \mathrm{d} s$ for all $0 \leq s \leq s^{\prime} \leq t$. Moreover $t \mapsto \mathfrak{S}(X)\left(s, s^{\prime} ; t\right)$ and $t \mapsto \mathfrak{S}(X)(s, t ; t)$ are continuous. If $X \in \mathcal{C}^{\gamma}\left([0, T] ; \mathcal{B}_{-\alpha}\right)$, then one can find a sequence $\left(X^{n}\right)_{n \in \mathbb{N}}$ such that $X^{n} \in \mathcal{C}^{1}\left([0, T] ; \mathcal{B}_{-\alpha}\right)$ and converging to $X$ in $\mathcal{C}^{\gamma^{\prime}}\left([0, T] ; \mathcal{B}_{-\alpha}\right)$ for all $\gamma^{\prime}<\gamma$

It follows from (12) and (13) that the speeds of convergence of

$$
\begin{aligned}
A^{\delta} \mathfrak{S}\left(X^{n}\right)\left(s, s^{\prime} ; t\right) & \rightarrow A^{\delta} \mathfrak{S}(X)\left(s, s^{\prime} ; t\right) \\
A^{\delta} \mathfrak{S}\left(X^{n}\right)(s, t ; t) & \rightarrow A^{\delta} \mathfrak{S}(X)(s, t ; t)
\end{aligned}
$$

in $\mathcal{C}^{\gamma^{\prime}}\left([0, T] ; \mathcal{B}_{\delta}\right)$ are uniform in $s \leq s^{\prime} \leq t$ in $[0, T]$ if $\alpha+\delta<\gamma^{\prime}$. Hence, we get that $t \mapsto \mathfrak{S}(X)\left(s, s^{\prime} ; t\right)$ and $t \mapsto \mathfrak{S}(X)(s, t ; t)$ are continuous in $\mathcal{B}_{\delta}$ for all $\delta>0$ such that $\alpha+\delta<\gamma$. 
- Hölder continuity of $\mathfrak{S}(X)$. By construction,

$$
\mathfrak{S}(X)\left(s, s^{\prime} ; t\right)+\mathfrak{S}(X)\left(s^{\prime}, s^{\prime \prime} ; t\right)=\mathfrak{S}(X)\left(s, s^{\prime \prime} ; t\right)
$$

for all $0 \leq s \leq s^{\prime} \leq s^{\prime \prime} \leq t$.

Fix $h>0$ and assume that $h=t \ell / 2^{m}$ for $\ell \in\left\{0, \ldots, 2^{m}\right\}$ and $m \in \mathbb{N}$. Then, by the semi-group property,

$$
\mathfrak{S}^{n}(0, t ; t+h)=S(h) \sum_{k=0}^{\left\lfloor 2^{n} t /(t+h)\right\rfloor-1} S\left(t-t_{k}^{n}\right) X\left(t_{k}^{n}, t_{k+1}^{n}\right) .
$$

As $h$ is a dyadic on $[0, t]$, we have $\left\lfloor 2^{n} t /(t+h)\right\rfloor=2^{n-m} \ell$. Passing to the limit in $\mathfrak{S}^{n}(i h,(i+1) h ; t)$ for $i \in\{0, \ldots, \ell-1\}$ and using (14) we obtain that

$$
\mathfrak{S}(X)(0, t ; t+h)=S(h) \mathfrak{S}(X)(0, t ; t) .
$$

In a similar way,

$$
\mathfrak{S}(X)(t, t+h ; t+h)=\mathfrak{S}\left(X_{t+}\right)(0, h ; h),
$$

where $X_{t+}$. is the path $\left(X_{t+s}\right)_{s \geq 0}$. Furthermore, the continuity of $h \mapsto$ $\mathfrak{S}(X)(0, t ; t+h)$ and $h \mapsto \mathfrak{S}(X)(0, h ; h)$ together with the continuity of $h \mapsto S(h) x$ for all $x \in \mathcal{B}$ implies that (15) and (16) are true even if $h$ is not a dyadic point of $[0, t]$.

Now,

$$
\begin{aligned}
& A^{\delta} \mathfrak{S}(X)(0, t+h ; t+h)-A^{\delta} \mathfrak{S}(X)(0, t ; t) \\
& \quad=A^{\delta} \mathfrak{S}(X)(0, t ; t+h)+A^{\delta} \mathfrak{S}(t, t+h ; t+h)-A^{\delta} \mathfrak{S}(X)(0, t ; t) \\
& =(S(h)-\mathrm{Id}) A^{\delta} \mathfrak{S}(X)(0, t ; t)+A^{\delta} \mathfrak{S}(t, t+h ; t+h) .
\end{aligned}
$$

Using (12) and (13), there exist some constants $K_{1}$ and $K_{2}$ depending only on $\alpha, \beta, \delta$ and $T$ such that

$$
\left\|A^{\delta} \mathfrak{S}(X)(0, t ; t)\right\| \leq K_{1} H(Y) t^{\gamma-\delta-\alpha}+\left\|A^{\delta+\alpha} S(t) Y(0, t)\right\| \leq K_{2} H(Y) t^{\gamma-\delta-\alpha}
$$

and that, invoking (16),

$$
\left\|A^{\delta} \mathfrak{S}(X)(t, t+h ; t+h)\right\| \leq K_{2} H(Y) h^{\gamma-\delta-\alpha} .
$$

Thus, for all $\beta$ in $(0,1]$ such that $\alpha+\beta+\delta<\gamma$,

$$
\begin{aligned}
\left\|(S(h)-\mathrm{Id}) A^{\delta} \mathfrak{S}(X)(0, t ; t)\right\| & \leq K_{\beta} h^{\beta}\left\|A^{\delta+\beta} \mathfrak{S}(t, t+h ; t+h)\right\| \\
& \leq K_{\beta} K_{2} H(Y) h^{\beta} t^{\gamma-\delta-\alpha}
\end{aligned}
$$

and hence

$$
\left\|A^{\delta} \mathfrak{S}(X)(0, t+h ; t+h)-A^{\delta} \mathfrak{S}(X)(0, t ; t)\right\| \leq K_{3} H(Y) h^{\beta}
$$

for all $t \in[0, T-h]$, where $K_{3}$ is a constant that depends only on $T$ and $\alpha$, $\beta, \gamma, \delta$. 


\section{The non-linear Cauchy problem}

We will now define and solve our evolution equation in the non-linear case: Let $\mathcal{U}$ be a separable Banach space, and $X$ a $\gamma$-Hölder continuous path with value in $\mathcal{U}$. Fix $\delta>0, \rho \in \mathbb{R}$ and let $B$ be a map from $\mathcal{B}_{\delta}$ to $\mathcal{L}\left(\mathcal{U} ; \mathcal{B}_{\rho}\right)$ (the space of linear bounded operators form $\mathcal{U}$ to $\mathcal{B}_{\rho}$ ).

Consider the non-linear Cauchy problem

$$
Y(t)=y+\int_{0}^{t} B(Y(s)) \mathrm{d} X(s)-\int_{0}^{t} A Y(s) \mathrm{d} s, \quad y \in \mathcal{B}_{\delta+\kappa}
$$

where the solution $Y$ is assumed to be $\kappa$-Hölder continuous with values in $\mathcal{B}_{\delta}$. The condition $y \in \mathcal{B}_{\delta+\kappa}$ on the initial condition is the natural one to ensure that the path $t \mapsto S(t) y$ belongs to $\mathcal{C}^{\kappa}\left([0, T], \mathcal{B}_{\delta}\right)$ for any $T>0$.

The integral with respect $\mathrm{d} X$ in the r.h.s. of eq.(17) is a Young integral which must be understood according to the following proposition:

Proposition 1. Let $\mathcal{V}$ be a Banach space and let $H \in \mathcal{C}^{\alpha}([0, T] ; L(\mathcal{U}, \mathcal{V}))$ with $\alpha+\gamma>1$. Then the integral

$$
F(t)=\int_{0}^{t} H(s) \mathrm{d} X(s)
$$

exists as the limit in $\mathcal{V}$ of the sums

$$
F^{\Pi}(t)=\sum_{\left\{s_{i}\right\}} H\left(s_{i}\right)\left[X\left(s_{i+1}\right)-X\left(s_{i}\right)\right]
$$

over the partitions $\Pi=\left\{s_{i}, i=0, \ldots, n: 0=s_{0} \leq s_{1} \cdots \leq s_{n}=t\right\}$ of the interval $[0, t]$ and as the size of the partition goes to zero. Moreover there exists a constant $K_{\alpha+\gamma}$ depending only on $\alpha+\gamma$ such that

$$
\begin{aligned}
& \|F(t)-F(s)-H(s)(X(t)-X(s))\|_{\mathcal{V}} \leq \\
& K_{\alpha+\gamma} H_{\gamma, T}(X ; \mathcal{U}) H_{\alpha, T}(H ; L(\mathcal{U}, \mathcal{V}))|t-s|^{\alpha+\gamma}
\end{aligned}
$$

for any $0 \leq s \leq t \leq T$.

Proof. This statement is a particular case of a more general one proved by Young (You36), see for example Lyons (Lvo98; LQ02).

We will prove that eq.(17) has a solution in the mild sense, i.e. we will prove that, under suitable assumptions, there exists a solution $Y$ to the equation

$$
Y(t)=S(t) y+\int_{0}^{t} S(t-s) B(Y(s)) \mathrm{d} X(s)
$$


which is the formal variation of constant solution to eq.(17).

Our approach to the construction of the solution is to recast eq.(201) as a fixed point problem for the application

$$
\Gamma: \mathcal{C}^{\kappa}\left([0, T], \mathcal{B}_{\delta}\right) \rightarrow \mathcal{C}^{\kappa}\left([0, T], \mathcal{B}_{\delta}\right)
$$

defined as

$$
\Gamma(Y)(t)=S(t) y+\int_{0}^{t} S(t-s) B(Y(s)) \mathrm{d} X(s)
$$

First we will show that $\Gamma$ maps a closed ball of $\mathcal{C}^{\kappa}\left([0, T], \mathcal{B}_{\delta}\right)$ into itself, and then, assuming a kind of Lipschitz condition for the operator $B$, we will show that $\Gamma$ is a contraction in this ball for a small time $\tau$ and obtain a unique solution up to $\tau$.

Hypothesis 1. Assume that there exists a function $C$ from $\mathcal{B}_{\delta}$ with values in $L\left(\mathcal{B}_{\delta} \otimes \mathcal{U}, \mathcal{B}_{\rho}\right)$ (where $\mathcal{B}_{\delta} \otimes \mathcal{U}$ is the tensor product endowed with the tensor norm, that is a norm $\|\cdot\|_{\mathcal{B}_{s} \otimes \mathcal{U}}$ such that $\|x \otimes y\| \leq\|x\|_{\mathcal{B}_{\delta}}\|y\|_{\mathcal{U}}$ for all $(x, y) \in$ $\mathcal{B}_{\delta} \times \mathcal{U}$ : see for example (LQ0\%), p. 144 for different possibilites for such a norm) such that

$$
B\left(y^{\prime}\right) x-B(y) x=\int_{0}^{1} C\left(y+\tau\left(y^{\prime}-y\right)\right)\left(y^{\prime}-y\right) \otimes x \mathrm{~d} \tau .
$$

for all $y, y^{\prime} \in \mathcal{B}_{\delta}$ and all $x \in \mathcal{U}$. Also assume that

$$
M_{B}(r)=\sup _{\|y\|_{\mathcal{B}_{\delta}} \leq r}\|C(y)\|_{L\left(\mathcal{B}_{\delta} \otimes \mathcal{U}, \mathcal{B}_{\rho}\right)}<\infty
$$

for any $r$ and that for some increasing function $M_{C}(r)>0, \epsilon \in(0,1]$ and for all $y, y^{\prime}, z \in \mathcal{B}_{\delta}$ such that $\|y\|_{\mathcal{B}_{\delta}} \leq r,\left\|y^{\prime}\right\|_{\mathcal{B}_{\delta}} \leq r$

$$
\left\|C\left(y^{\prime}\right) z \otimes x-C(y) z \otimes x\right\|_{\mathcal{B}_{\rho}} \leq M_{C}(r)\left\|y^{\prime}-y\right\|_{\mathcal{B}_{\delta}}^{\epsilon}\|z\|_{\mathcal{B}_{\delta}}\|x\| .
$$

for any $x$ in $\mathcal{U}$.

Lemma 1. Under the previous assumptions on $X$ and Hypothesis 1, if

$$
\gamma+\kappa>1 \text { and } 0<\kappa<\max \{\gamma+\rho-\delta, 1\}
$$

then there exists $R>0$ and $\tau>0$ such that the closed subset of $\mathcal{C}^{\kappa}\left([0, \tau] ; \mathcal{B}_{\delta}\right)$ defined by

$$
\begin{aligned}
& \mathcal{W}_{\tau}(y, R) \\
& \quad:=\left\{x \in \mathcal{C}^{\kappa}\left([0, \tau] ; \mathcal{B}_{\delta}\right) \mid x(0)=y ; H_{\kappa, \tau}(x) \leq R, \sup _{s \in[0, \tau]}\|x(s)\|_{\mathcal{B}_{\delta+\kappa}} \leq R\right\} .
\end{aligned}
$$


is invariant under $\Gamma$. Moreover $\tau$ can be chosen independently of $y$ if

$$
\sup _{r>0} M_{B}(r)<\infty .
$$

Proof. Take $Y \in \mathcal{W}_{\tau}(y, R)$, and note that

$$
\sup _{s \in[0, \tau]}\|Y(s)\|_{\mathcal{B}_{\delta}} \leq\|y\|_{\mathcal{B}_{\delta}}+R \tau^{\kappa}=\tilde{R} .
$$

In order to estimate $\Gamma(Y)$ we will in two steps. First we write

$$
\Gamma(Y)(t)=S(t) y+\int_{0}^{t} S(t-s) \mathrm{d} Z_{s}
$$

where $Z$ is given by

$$
Z(t):=\int_{0}^{t} B(Y(s)) \mathrm{d} X(s)
$$

Then, Proposition 1 and the assumptions on $B$ imply that $Z$ is $\gamma$-Hölder continuous with values in $\mathcal{B}_{\rho}$, and that there exists some constant $K$ depending only on the parameters such that for all $0 \leq s \leq t \leq \tau$,

$$
\begin{aligned}
\left\|Z^{n}(t)-Z^{n}(s)-B\left(Y^{n}(s)\right)(X(t)-X(s))\right\|_{\mathcal{B}_{\rho}} \\
\quad \leq K M_{B}(\tilde{R}) H_{\gamma, \tau}(X ; \mathcal{U}) H_{\kappa, \tau}\left(Y ; \mathcal{B}_{\delta}\right)|t-s|^{\gamma+\kappa} .
\end{aligned}
$$

This in turn imply that $\Gamma(Y)$ is given by (24). Indeed from the assumption that $\gamma+\kappa>1$ it follows easily that

$$
\int_{0}^{t} S(t-s) B(Y(s)) \mathrm{d} X(s)=\int_{0}^{t} S(t-s) \mathrm{d} Z(s),
$$

since $\sum_{i=0}^{k-1}\left(t_{i+1}-t_{i}\right)^{\gamma+\kappa}$ converges to 0 with the mesh of the partition $0 \leq$ $t_{0} \leq \cdots \leq t_{k} \leq t$.

The estimate (25) can be elaborated further to give

$$
\begin{array}{r}
\|Z(t)-Z(s)\|_{\mathcal{B}_{\rho}} \leq H_{\gamma, T}(X ; \mathcal{U})\left(\|B(y)\|_{\mathcal{U} \rightarrow \mathcal{B}_{\rho}}+M_{B}(\tilde{R}) H_{\kappa, \tau}\left(Y ; \mathcal{B}_{\delta}\right) \tau^{\kappa}\right)|t-s|^{\gamma} \\
+K M_{B}(\tilde{R}) H_{\gamma, T}(X ; \mathcal{U}) H_{\kappa, \tau}\left(Y ; \mathcal{B}_{\delta}\right)|t-s|^{\gamma+\kappa}
\end{array}
$$


where we used the following inequality to bound the supremum of $B(Y(s))$ :

$$
\begin{aligned}
\sup _{s \in[0, \tau]}\|B(Y(s))\|_{\mathcal{U} \rightarrow \mathcal{B}_{\rho}} & \leq\|B(y)\|_{\mathcal{U} \rightarrow \mathcal{B}_{\rho}}+\sup _{s \in[0, \tau]}\|B(Y(s))-B(Y(0))\|_{\mathcal{U} \rightarrow \mathcal{B}_{\rho}} \\
& \leq\|B(y)\|_{\mathcal{U} \rightarrow \mathcal{B}_{\rho}}+\sup _{s, t \in[0, \tau]}\|B(Y(s))-B(Y(t))\|_{\mathcal{U} \rightarrow \mathcal{B}_{\rho}} \\
& \leq\|B(y)\|_{\mathcal{U} \rightarrow \mathcal{B}_{\rho}}+M_{B}(\tilde{R}) \sup _{s, t \in[0, \tau]}\|Y(s)-Y(t)\| \\
& \leq\|B(y)\|_{\mathcal{U} \rightarrow \mathcal{B}_{\rho}}+M_{B}(\tilde{R}) \tau^{\kappa}\left(\sup _{s, t \in[0, \tau]} \frac{\|Y(s)-Y(t)\|}{|t-s|^{\kappa}}\right) \\
& =\|B(y)\|_{\mathcal{U} \rightarrow \mathcal{B}_{\rho}}+M_{B}(\tilde{R}) H_{\kappa, \tau}\left(Y ; \mathcal{B}_{\delta}\right) \tau^{\kappa} .
\end{aligned}
$$

Note that

$$
H_{\kappa, \tau}\left(S(\cdot) y ; \mathcal{B}_{\delta}\right) \leq C_{\kappa}\|y\|_{\mathcal{B}_{\delta+\kappa}} .
$$

Thus, it follows from (10) that for some constant $C$ (that does not depend on $Y$ ), we have

$$
\begin{aligned}
& H_{\kappa, \tau}\left(\Gamma(Y) ; \mathcal{B}_{\delta}\right) \leq C_{\kappa}\|y\|_{\mathcal{B}_{\delta+\kappa}} \\
& \quad+C H_{\gamma, T}(X ; \mathcal{U})\left(\|B(y)\|_{\mathcal{U} \rightarrow \mathcal{B}_{\rho}}+(1+K) M_{B}(\tilde{R}) H_{\kappa, \tau}\left(Y ; \mathcal{B}_{\delta}\right) \tau^{\kappa}\right) .
\end{aligned}
$$

By similar arguments, and using eq.(11), we obtain also

$$
\begin{aligned}
\sup _{s \in[0, \tau]} & \|\Gamma(Y)(s)\|_{\mathcal{B}_{\delta+\kappa}} \leq\|y\|_{\mathcal{B}_{\delta+\kappa}} \\
& +C H_{\gamma, T}(X ; \mathcal{U})\left(\|B(y)\|_{\mathcal{U} \rightarrow \mathcal{B}_{\rho}}+(1+K) M_{B}(\tilde{R}) H_{\kappa, \tau}\left(Y ; \mathcal{B}_{\delta}\right) \tau^{\kappa}\right)
\end{aligned}
$$

where we assumed that $\tau \leq 1$ to obtain an expression similar to (26). Choose now $\theta \in(0,1)$ and set

$$
R=(1-\theta)^{-1}\left[C_{\kappa}\|y\|_{\mathcal{B}_{\delta+\kappa}}+C H_{\gamma, T}(X ; \mathcal{U})\|B(y)\|_{\mathcal{U} \rightarrow \mathcal{B}_{\rho}}\right]
$$

and $\tau>0$ such that

$$
M_{B}\left(\|y\|_{\mathcal{B}_{\delta}}+R \tau^{\kappa}\right) \tau^{\kappa} \leq \theta\left[(1+K) C H_{\gamma, T}(X ; \mathcal{U})\right]^{-1} .
$$

With this choice of $\tau, R$ the left-hand side in (26) is smaller than $R$. This implies that $\Gamma(Y)$ belongs to $\mathcal{W}_{\tau}(y, R)$ if $Y$ belongs to $\mathcal{W}_{\tau}(y, R)$.

Moreover, note that if we set

$$
M_{B}=\sup _{r \geq 0} M_{B}(r)<\infty
$$


then the choice

$$
\tau^{\kappa}=\frac{\theta}{(1+K) M_{B} C H_{\gamma, T}(X ; \mathcal{U})} .
$$

is equally good and independent of $y$.

Theorem 2. Under Hypothesis 1 for $B$ and conditions (23) for $\delta$ and $\kappa$, there exists a time $T>0$ up to which a unique solution $Y$ of eq.(20) in $\mathcal{C}^{\kappa}\left([0, T] ; \mathcal{B}_{\delta}\right)$ exists. If $M_{B}<\infty, T$ can be chosen arbitrarily large. Moreover the map $X \mapsto Y$ is Lipschitz continuous from $\mathcal{C}^{\gamma}([0, T] ; \mathcal{U})$ to $\mathcal{C}^{\kappa}\left([0, T] ; \mathcal{B}_{\delta}\right)$.

Proof. Choose $\tilde{\tau}, R$ according to Lemma 1 in order to have $\Gamma: \mathcal{W}_{\tilde{\tau}}(y, R) \rightarrow$ $\mathcal{W}_{\tilde{\tau}}(y, R)$. Our aim is to show that there exists $\tau \in(0, \tilde{\tau}]$ such that $\Gamma$ is a strict contraction in $\mathcal{W}_{\tau}(y, R) \subseteq \mathcal{W}_{\tilde{\tau}}(y, R)$.

Let $Y$ and $Y^{\prime}$ be two paths in $\mathcal{W}_{\tau}(y, R)$. Set

$$
Z(t)=\int_{0}^{t} B(Y(s)) \mathrm{d} X(s) \text { and } Z^{\prime}(t)=\int_{0}^{t} B\left(Y^{\prime}(s)\right) \mathrm{d} X(s) .
$$

By Proposition 1 we have the estimate

$$
\begin{aligned}
& \left\|Z(t)-Z^{\prime}(t)-Z(s)-Z^{\prime}(s)\right\|_{\mathcal{B}_{\rho}} \\
& \quad \leq K H_{\gamma, \tau}(X ; \mathcal{U})\left(\sup _{u \in[0, \tau]}\left\|B(Y(u))-B\left(Y^{\prime}(u)\right)\right\|_{L\left(\mathcal{U}, \mathcal{B}_{\rho}\right)}|t-s|^{\gamma}\right. \\
& \left.\quad+H_{\kappa \epsilon, \tau}\left(B(Y(\cdot))-B\left(Y^{\prime}(\cdot)\right) ; L\left(\mathcal{U}, \mathcal{B}_{\rho}\right)\right)|t-s|^{\gamma+\kappa \epsilon}\right)
\end{aligned}
$$

for any $s, t \in[0, \tau]$. Then,

$$
\begin{gathered}
\sup _{u \in[0, \tau]}\left\|B(Y(u))-B\left(Y^{\prime}(u)\right)\right\|_{L\left(\mathcal{U}, \mathcal{B}_{\rho}\right)} \leq M_{B}(\tilde{R}) \sup _{u \in[0, \tau]}\left\|Y(u)-Y^{\prime}(u)\right\|_{\mathcal{B}_{\rho}} \\
\leq M_{B}(\tilde{R}) \sup _{s, t \in[0, \tau]}\left\|Y(s)-Y^{\prime}(s)-Y(t)+Y^{\prime}(t)\right\|_{\mathcal{B}_{\rho}} \\
\leq M_{B}(\tilde{R}) H_{\kappa, \tau}\left(Y(s)-Y^{\prime}(s) ; \mathcal{B}_{\rho}\right) \tau^{\kappa}
\end{gathered}
$$

Moreover, calling $\Delta=Y-Y^{\prime}$ we have

$$
\begin{aligned}
B\left(Y^{\prime}(t)\right) x- & B(Y(t)) x-B\left(Y^{\prime}(s)\right) x+B(Y(s)) x= \\
= & \int_{0}^{1} C(Y(t)+r \Delta(t)) \mathrm{d} r \Delta(t) \otimes x \\
& -\int_{0}^{1} C(Y(s)+r \Delta(s)) \mathrm{d} r \Delta(s) \otimes x \\
= & \int_{0}^{1}[C(Y(t)+r \Delta(t))-C(Y(s)+r \Delta(s))] \mathrm{d} r \Delta(t) \otimes x \\
& +\int_{0}^{1} C(Y(s)+r \Delta(s)) \mathrm{d} r[\Delta(t)-\Delta(s)] \otimes x
\end{aligned}
$$


which yields the following estimate:

$$
\begin{aligned}
& \left\|B(Y(t))-B\left(Y^{\prime}(t)\right)-B(Y(s))-B\left(Y^{\prime}(s)\right)\right\|_{L\left(\mathcal{U}, \mathcal{B}_{\rho}\right)} \leq \\
& \quad 3 M_{C}(\tilde{R})\left(H_{\kappa, \tau}\left(Y ; \mathcal{B}_{\delta}\right)+H_{\kappa, \tau}\left(Y^{\prime} ; \mathcal{B}_{\delta}\right)\right)^{\epsilon} \sup _{s \in[0, \tau]}\left\|Y(s)-Y^{\prime}(s)\right\|_{\mathcal{B}_{\delta}}|t-s|^{\kappa \epsilon} \\
& \quad+M_{B}(\tilde{R}) H_{\kappa, \tau}\left(Y-Y^{\prime} ; \mathcal{B}_{\delta}\right)|t-s|^{\kappa} \\
& \quad \leq H_{\kappa, \tau}\left(Y-Y^{\prime} ; \mathcal{B}_{\delta}\right)\left(3 M_{C}(\tilde{R})(2 R)^{\epsilon} \tau^{\kappa}+M_{B}(\tilde{R}) \tau^{(1-\epsilon) \kappa}\right)|t-s|^{\kappa \epsilon} .
\end{aligned}
$$

Thus

$$
H_{\gamma, \tau}\left(Z-Z^{\prime} ; \mathcal{B}_{\rho}\right) \leq K_{\tau}^{\prime} H_{\kappa, \tau}\left(Y-Y^{\prime} ; \mathcal{B}_{\delta}\right)
$$

with

$$
K_{\tau}^{\prime}=K_{\gamma+\kappa \epsilon} H_{\gamma, T}(X ; \mathcal{U})\left(3 M_{C}(\tilde{R})(2 R)^{\epsilon} \tau^{\kappa}+M_{B}(\tilde{R}) \tau^{(1-\epsilon) \kappa}+M_{B}(\tilde{R}) \tau^{\kappa}\right)
$$

going to zero as $\tau \rightarrow 0$.

Thanks to Theorem 1, it follows that there exists a constant $K_{\tau}^{\prime \prime}$ decreasing to 0 with $\tau$ such that

$$
H_{\gamma, \tau}\left(\Gamma(Y)-\Gamma\left(Y^{\prime}\right) ; \mathcal{B}_{\delta}\right) \leq K_{\tau}^{\prime \prime} H_{\kappa, \tau}\left(Y^{\prime}-Y ; \mathcal{B}_{\delta}\right)
$$

and

$$
\sup _{s \in[0, \tau]}\left\|\Gamma(Y)(s)-\Gamma\left(Y^{\prime}\right)(s)\right\|_{\mathcal{B}_{\delta+\kappa}} \leq K_{\tau}^{\prime \prime} H_{\kappa, \tau}\left(Y^{\prime}-Y ; \mathcal{B}_{\delta}\right) .
$$

Thus, if $T$ is small enough, the map $Y \mapsto \Gamma(Y)$ is $K_{\tau}^{\prime \prime}$-Lipschitz on some given ball $\mathcal{W}_{\tau}(y, R)$ with $K_{\tau}^{\prime \prime}<1$. The uniqueness of the solution of eq.(20) on $[0, T]$ for $T \leq \tau$ follows immediately. Furthermore, in the case where $M_{B}<\infty$ the independence of $\tilde{\tau}$ from the starting point $y$ allows to prove iteratively the uniqueness for any small time interval $[i \tau,(i+1) \tau]$ for $i \leq\lfloor T / \tau\rfloor$ and thus deduce existence and uniqueness of the solution on an arbitrary time interval $[0, T]$.

The Lipschitz continuity of the map $X \mapsto Y$, where $Y$ is the solution of the Cauchy problem, can be proven along the following lines: let $Y$ the solution of the evolution equation driven by $X$ and $Y^{\prime}$ that driven by another path $X^{\prime} \in \mathcal{C}^{\gamma}([0, T], \mathcal{U})$, and set

$$
Z(t)=\int_{0}^{t} B(Y(s)) \mathrm{d} X(s), \quad Z^{\prime}(t)=\int_{0}^{t} B\left(Y^{\prime}(s)\right) \mathrm{d} X^{\prime}(s),
$$

We can decompose $Z-Z^{\prime}$ as

$$
\begin{aligned}
Z(t)-Z^{\prime}(t)=\int_{0}^{t}[B(Y(s)) & \left.-B\left(Y^{\prime}(s)\right)\right] \mathrm{d} X(s) \\
& +\int_{0}^{t} B(Y(s)) \mathrm{d}\left[X(s)-X^{\prime}(s)\right]=\Delta^{1}(t)+\Delta^{2}(t),
\end{aligned}
$$


noting that all the Young integrals are well defined. Then

$$
\begin{aligned}
& Y(t)-Y^{\prime}(t)=\int_{0}^{t} S(t-s) d {\left[Z(s)-Z^{\prime}(s)\right] } \\
&=\int_{0}^{t} S(t-s) \mathrm{d} \Delta^{1}(s)+\int_{0}^{t} S(t-s) \mathrm{d} \Delta^{2}(s) .
\end{aligned}
$$

Using now the same kind of estimates as in the beginning of this poof, we obtain a bound of the form

$$
H_{\gamma, \tau}\left(Y-Y^{\prime} ; \mathcal{B}_{\delta}\right) \leq K_{\tau}^{\prime \prime \prime} H_{\gamma, \tau}\left(Y-Y^{\prime} ; \mathcal{B}_{\delta}\right)+C H_{\gamma, \tau}\left(X-X^{\prime} ; \mathcal{U}\right)
$$

where $K_{\tau}^{\prime \prime \prime}$ goes to zero as $\tau$ goes to zero. So for a small time interval $\tau$ we obtain

$$
H_{\gamma, \tau}\left(Y-Y^{\prime} ; \mathcal{B}_{\delta}\right) \leq C^{\prime} H_{\gamma, \tau}\left(X-X^{\prime} ; \mathcal{U}\right)
$$

Next, some simple argument on Hölder norms (see e.g. (Gub04)) can be used to show that this estimate is true up to the existence time $T$ of the solution:

$$
H_{\gamma, T}\left(Y-Y^{\prime} ; \mathcal{B}_{\delta}\right) \leq C^{\prime} H_{\gamma, T}\left(X-X^{\prime} ; \mathcal{U}\right)
$$

Remark 1. The results of Theorems 1 and 2 are still true when (17) is replaced by

$$
Y(t)=y+\int_{0}^{t} B(Y(s)) \mathrm{d} X(s)+\int_{0}^{t} F(Y(s)) \mathrm{d} s-\int_{0}^{t} A Y(s) \mathrm{d} Y(s),
$$

where $X$ belongs to $\mathcal{C}^{\gamma}([0, T] ; \mathcal{U})$, and where $F$ is a Lipschitz function from $\mathcal{B}_{\delta}$ to $\mathcal{B}_{\rho}$.

\section{The stochastic heat equation in one dimen- sion}

In this section, we focus our attention on the (formal) equation

$$
\partial_{t} Y(t, x)=\Delta Y(t, x)+\sigma(Y(t, x)) \mathrm{d} X_{t}(x), \quad t \in[0, T], x \in[0,1],
$$

with Dirichlet boundary conditions and a null initial condition and we try to find some simple assumptions on the noise $X$ and on the function $\sigma: \mathbb{R} \rightarrow \mathbb{R}$ ensuring the existence and uniqueness of a mild solution to (133) in the sense of Theorems 1 and 2 Of course our abstract setting could be applied to 
some more general equations, but the heat equation in dimension one is a good example to see if our general result can be used in a simple case, and we are also able to compare it with the abundant existing literature on this equation.

To get started, let us describe the kind of noise we consider.

\subsection{Fractional Brownian noise}

Consider $\left\{e_{n} ; n \geq 1\right\}$ the trigonometrical basis of $L_{0}^{2}([0,1])$, that is

$$
e_{n}(x)=\left(\frac{\pi}{2}\right)^{1 / 2} \sin (2 \pi n x), \quad n \geq 1, x \in[0,1]
$$

and a collection $\left\{\hat{W}^{n} ; n \geq 1\right\}$ of independent fractional Brownian motions with Hurst parameter $H \in(0,1)$, all defined on the same probability space $(\Omega, \mathcal{F}, P)$. Recall that $\hat{W}^{n}$ is then a centered Gaussian process with covariance

$$
E\left[\hat{W}^{n}(s) \hat{W}^{n}(t)\right]=\frac{1}{2}\left(t^{2 H}+s^{2 H}-|t-s|^{2 H}\right), \quad s, t \in[0, T] .
$$

In the sequel, the spaces $\mathcal{B}_{\alpha}$ is also identified with the usual Sobolev spaces $\mathcal{W}_{0}^{2 \alpha, p}$ for $\alpha>0$ and a fixed $p \geq 1$. When $\zeta \in(0,1), \mathcal{W}_{0}^{\zeta, p}$ can be defined by the completion of the smooth functions on $[0,1]$ with Dirichlet boundary conditions, with respect to the norm

$$
\|\varphi\|_{\zeta, p}^{p}=|\varphi|_{p}^{p}+\int_{[0,1]^{2}} \frac{|\varphi(x)-\varphi(y)|^{p}}{|x-y|^{1+\zeta p}} \mathrm{~d} x \mathrm{~d} y .
$$

Recall that for two conjugate exponents $p, q$ and $\zeta \in(0,1), \mathcal{W}_{0}^{-\zeta, q}$ is defined as the dual space, in the distribution sense, of $\mathcal{W}_{0}^{\zeta, p}$. Recall also that for any $\alpha, \zeta>0$, the operator $(-\Delta)^{\alpha}: \mathcal{W}_{0}^{\zeta, p} \rightarrow \mathcal{W}_{0}^{\zeta-2 \alpha, p}$, is one-to-one. Then, we can identify $\mathcal{B}_{-\alpha}$ with $\mathcal{W}_{0}^{-2 \alpha, p}$ when $\alpha>0$.

With these notations in mind, a Gaussian Hölder continuous process with values in a Sobolev space of negative order can easily be constructed:

Lemma 2. Let $T, \mu$ be two positive real numbers with $\mu \in(0,1)$, and $\left\{q_{n} ; n \geq\right.$ 1) a collection of positive numbers such that

$$
\sum_{n \geq 1}\left(\frac{q_{n}}{n^{\mu}}\right)^{2}<\infty
$$


Consider the random field $X$ defined by the formal series

$$
X(t, x)=\sum_{n \geq 1} q_{n} e_{n}(x) \hat{W}^{n}(t), \quad t \in[0, T], x \in[0,1] .
$$

Then, for any $\gamma<H, \mu<\alpha<1, \hat{p} \geq 1$, we have $X \in \mathcal{C}^{\gamma}\left([0, T], \mathcal{W}_{0}^{-\alpha, \hat{p}}\right)$ almost surely.

Proof. Let $\gamma<H, \alpha>\mu$, and $\nu$ be a real number such that $\zeta \equiv 2 \nu-\alpha>0$. Since

$$
(-\Delta)^{-\nu}: \mathcal{W}_{0}^{-\alpha, \hat{p}} \rightarrow \mathcal{W}_{0}^{\zeta, \hat{p}}
$$

is a one-to-one operator, it is enough to see that the process $V=(-\Delta)^{-\nu} X$ is almost surely an element of $\mathcal{C}^{\gamma}\left([0, T], \mathcal{W}_{0}^{\zeta, \hat{p}}\right)$. However, $V$ is defined by the series

$$
V(t, x)=\sum_{n \geq 1} v_{n} e_{n}(x) \hat{W}^{n}(t), \quad t \in[0, T], x \in[0,1]
$$

with

$$
v_{n}=\frac{c_{1} q_{n}}{n^{2 \nu}} \quad \text { and } \quad c_{1}=\frac{1}{(2 \pi)^{2 \nu}} .
$$

Now, if $t_{1}, t_{2} \in[0, T]$ and $x_{1}, x_{2} \in[0,1]$, we have

$$
\begin{aligned}
E\left[\left|V\left(t_{2}, x_{2}\right)-V\left(t_{1}, x_{1}\right)\right|^{2}\right] \leq 2(E[ & \left.\left|V\left(t_{2}, x_{2}\right)-V\left(t_{1}, x_{2}\right)\right|^{2}\right] \\
& \left.+E\left[\left|V\left(t_{1}, x_{2}\right)-V\left(t_{1}, x_{1}\right)\right|^{2}\right]\right) .
\end{aligned}
$$

Furthermore,

$$
\begin{array}{r}
E\left[\left|V\left(t_{2}, x_{2}\right)-V\left(t_{1}, x_{2}\right)\right|^{2}\right]=E\left[\left(\sum_{n \geq 1} v_{n} e_{n}\left(x_{2}\right)\left(\hat{W}^{n}\left(t_{2}\right)-\hat{W}^{n}\left(t_{1}\right)\right)\right)^{2}\right] \\
\leq\left(\sum_{n \geq 1} v_{n}^{2} e_{n}^{2}\left(x_{2}\right)\right)\left|t_{2}-t_{1}\right|^{2 H} \leq c\left(\sum_{n \geq 1} v_{n}^{2}\right)\left|t_{2}-t_{1}\right|^{2 H} .
\end{array}
$$

Now, since $2 \nu-\mu>0$, it is easily seen from condition (34) that

$$
E\left[\left|V\left(t_{2}, x_{2}\right)-V\left(t_{1}, x_{2}\right)\right|^{2}\right] \leq c\left|t_{2}-t_{1}\right|^{2 H} \text {. }
$$

On the other hand,

$$
\begin{aligned}
& E\left[\left|V\left(t_{1}, x_{2}\right)-V\left(t_{1}, x_{1}\right)\right|^{2}\right]=E {\left[\left(\sum_{n \geq 1} v_{n}\left(e_{n}\left(x_{2}\right)-e_{n}\left(x_{1}\right)\right) \hat{W}^{n}\left(t_{1}\right)\right)^{2}\right] } \\
& \leq c T^{2 H}\left(\sum_{n \geq 1} n^{2(2 \nu-\mu)} v_{n}^{2}\right)\left|x_{2}-x_{1}\right|^{2(2 \nu-\mu)} .
\end{aligned}
$$


However,

$$
\sum_{n \geq 1} n^{2(2 \nu-\mu)} v_{n}^{2}=c \sum_{n \geq 1}\left(\frac{q_{n}}{n^{\mu}}\right)^{2}<\infty
$$

and thus

$$
E\left[\left|V\left(t_{1}, x_{2}\right)-V\left(t_{1}, x_{1}\right)\right|^{2}\right] \leq c\left|x_{2}-x_{1}\right|^{2(2 \nu-\mu)}
$$

Plugging (36) and (37) into (35), we get

$$
E\left[\left|V\left(t_{2}, x_{2}\right)-V\left(t_{1}, x_{1}\right)\right|^{2}\right] \leq c\left(\left|t_{2}-t_{1}\right|^{2 H}+\left|x_{2}-x_{1}\right|^{2(2 \nu-\mu)}\right),
$$

and since $V$ is a centered Gaussian process, this yields, by a simple application of Kolmogorov's criterion, that $V$ is almost surely Hölder continuous, with exponent $\gamma$ in time and $\zeta$ in space (recall that $\zeta=2 \nu-\alpha<2 \nu-\mu$ ). It is now easily seen that almost surely, $V \in \mathcal{C}^{\gamma}\left([0, T], \mathcal{W}_{0}^{\zeta, \hat{p}}\right)$, for any $\hat{p} \geq 1$.

\subsection{The linear case}

In this section, we just see how to read Theorem 11in our fractional Brownian context, in order to compare it with the existing results (see e.g. (TTV03)).

Proposition 2. Let $T$ be a positive constant, and $p \geq 1$. Assume that $X$ is a fractional Brownian noise defined as in Lemma 2, with $\mu<2 H$. Then there exists a unique mild solution to the equation

$$
\partial_{t} Y(t, x)=\Delta Y(t, x) \mathrm{d} t+\mathrm{d} X_{t}(x), \quad t \in[0, T], x \in[0,1],
$$

living in the space $\mathcal{C}^{\kappa}\left([0, T], \mathcal{W}_{0}^{\delta, p}\right)$ for all $\delta<2 H-\mu, 2 \kappa<2 H-\mu-\delta$.

Proof. This is a trivial consequence of Lemma 2 and Theorem 1 .

Remark 2. In the context of Proposition 2 the minimal condition under which (38) has a function-valued solution is morally $\sum_{n \geq 1} q_{n}^{2} n^{-4 H}<\infty$, which is the same necessary and sufficient condition as the one found by Tudor, Tindel and Viens (TTV03) in order to have solutions for the linear Cauchy problem. This tells us that the path-wise method reaches optimality in the case of time Hölder regularity greater than $1 / 2$, which is a kind of surprise, since the methods of (TTV03) rely on the isometric properties associated with the Gaussian stochastic integrals which are not exploited in the path-wise approach. Moreover we can handle a much more general class of noises. It is also worth noticing that, for any $H>1 / 2$, the coefficients $q_{n}=1$ (that is $\mu=1 / 2+\varepsilon$ for any $\varepsilon>0$ ) are consistent with the assumptions of our proposition, which means that the white noise in space, considered e.g. by Da Prato-Zabczyk (DPZ92) and Walsh (Wal86), can also be considered in our setting for the heat equation in dimension 1. 


\subsection{The non-linear Cauchy problem}

In this section, we take up the program of giving a simple enough example of an equation of the type introduced in eq. (33), admitting a function-valued solution. We get the following Theorem.

Theorem 3. Let $X$ be a noise defined like in Lemma Q and $\sigma \in \mathcal{C}_{b}^{2}(\mathbb{R})$. Suppose that $H>1 / 2$ and $\mu>0$ satisfy

There exist $\delta, \kappa \in(0,1)$ such that $2 \kappa<2 H-\mu-\delta$ and $H+\kappa>1$.

Then, there exists a unique mild solution to (33) in $\mathcal{C}^{\kappa}\left([0, T], \mathcal{W}_{0}^{\delta, p}\right)$, up to an explosion time $T$, for any $p \geq 1 / \delta$.

Proof. If (39) holds true, one can always find $\mu<\alpha<1$ such that $2 \kappa<$ $2 H-\alpha-\delta$ and $H+\kappa>1$. In fact, we will prove that the solution $Y_{t}$ to (33) lives in $\mathcal{W}_{0}^{\delta, p}$ for $p$ large enough: according to Lemma 2, recall that $X \in \mathcal{C}^{\gamma}\left([0, T], \mathcal{W}_{0}^{-\alpha, \hat{p}}\right)$ for any $\gamma<H, \alpha>\mu$ and $\hat{p} \geq 1$. Thus, going back to Theorem 2, we will first check that the operator-valued functional, defined on test functions $u, \varphi \in \mathcal{C}_{0}^{\infty}([0,1])$ by

$$
[B(u) \varphi](t, x)=\sigma(u(t, x)) \varphi(t, x)
$$

can be extended into an application

$$
B: \mathcal{W}_{0}^{\delta, p} \rightarrow \mathcal{L}\left(\mathcal{W}_{0}^{-\alpha, \hat{p}} ; \mathcal{W}_{0}^{-\alpha, p}\right)
$$

for $p$ large enough, and that this application satisfies Hypothesis 11. This will be done in two steps.

Step 1: If $\varphi \in \mathcal{W}_{0}^{-\alpha, \hat{p}}, B(u) \varphi$ is defined by duality: if $\hat{q}$ is the conjugate of $\hat{p}$, we set

$$
\mathcal{W}_{0}^{-\alpha, \hat{p}}(B(u) \varphi ; \psi)_{\mathcal{W}_{0}^{\alpha, \hat{q}}}=\mathcal{W}_{0}^{-\alpha, \hat{p}}(\varphi ; B(u) \psi)_{\mathcal{W}_{0}^{\alpha, \hat{q}}}
$$

Hence, a first condition in order to define $B$ properly is, for any $t \in[0, T]$, that

$$
\sigma(u(t, .)) \in \mathcal{W}_{0}^{\alpha, \hat{q}} \text { and } \mathcal{W}_{0}^{\alpha, \hat{q}} \text { is an algebra. }
$$

Now, if $u(t,.) \in \mathcal{W}_{0}^{\alpha, \hat{q}}$ and $\sigma \in \mathcal{C}_{b}^{2}(\mathbb{R}), \sigma(u(t,)$.$) is still an element of \mathcal{W}_{0}^{\alpha, \hat{q}}$, and thanks to the classical Sobolev imbeddings (see e.g. (Ada75)), if we assume that $u(t,.) \in \mathcal{W}_{0}^{\delta, p}$, the condition (40) is induced by

$$
\frac{1}{p}<\frac{1}{\hat{q}}+\delta-\alpha \quad \text { and } \quad \alpha \hat{q}>1
$$


and it is readily checked that this condition is equivalent to

$$
\hat{p}<\frac{1}{1-\alpha} \text { and } p>\frac{1}{\delta}
$$

Furthermore, we have seen that $\hat{p}$ (and hence $\hat{q}$ ) can be chosen arbitrarily, and thus, given $\alpha$ and $\delta$, one can always find $p$ and $\hat{p}$ satisfying condition (41). We assume that these coefficients have been chosen and fixed in the remainder of the proof.

Step 2: Let us check now Hypothesis [1] we use again a duality argument, and hence, it is enough to show that, if $u, v \in \mathcal{W}_{0}^{\delta, p}$, and $\psi \in \mathcal{W}_{0}^{\alpha, \hat{q}}$, then

$$
\|[B(u)-B(v)] \psi\|_{\mathcal{W}_{0}^{\alpha, \hat{q}}}=\int_{0}^{1}[D(u+\tau(v-u))](v-u) \otimes \psi \mathrm{d} \tau,
$$

with

$$
\|D(h)\|_{\mathcal{L}\left(\mathcal{W}_{0}^{\delta, p} \otimes \mathcal{W}_{0}^{\alpha, \hat{q}} ; \mathcal{W}_{0}^{\alpha, \hat{q}}\right)} \leq c\left(1+\|h\|_{\mathcal{W}_{0}^{\delta, p}}\right) .
$$

However, we have been reduced to a situation where $\mathcal{W}_{0}^{\delta, p}$ is continuously imbedded into $\mathcal{W}_{0}^{\alpha, \hat{q}}$, and thus, inequality (42) is implied by

$$
\|[D(h)] w \otimes \psi\|_{\mathcal{W}_{0}^{\alpha, \hat{q}}} \leq c\|\psi\|_{\mathcal{W}_{0}^{\alpha, \hat{q}}}\left(1+\|h\|_{\mathcal{W}_{0}^{\alpha, \hat{q}}}\right)\|w\|_{\mathcal{W}_{0}^{\alpha, \hat{q}}}
$$

which is the condition we check now: observe first that, from the definition of $B$, we have, for $x \in[0,1]$,

$$
[D(h) w \otimes \psi](x)=\sigma^{\prime}(h(x)) w(x) \psi(x) .
$$

Thus, the following decomposition holds true:

$$
\|[D(h)] w \otimes \psi\|_{\mathcal{W}_{0}^{\alpha, \hat{q}}} \leq J_{1}+J_{2}+J_{3}+J_{4},
$$

with

$$
\begin{aligned}
& J_{1}=\left\|\sigma^{\prime}(h) w \psi\right\|_{L^{q}} \\
& J_{2}=\int_{[0,1]^{2}} \frac{\left|\left[\sigma^{\prime}(h(x))-\sigma^{\prime}(h(y))\right] w(x) \psi(x)\right|}{|x-y|^{1+\alpha \hat{q}}} \mathrm{~d} x \mathrm{~d} y \\
& J_{3}=\int_{[0,1]^{2}} \frac{\left|(w(x)-w(y)) \sigma^{\prime}(h(y)) \psi(x)\right|}{|x-y|^{1+\alpha \hat{q}}} \mathrm{~d} x \mathrm{~d} y \\
& J_{4}=\int_{[0,1]^{2}} \frac{\left|(\psi(x)-\psi(y)) \sigma^{\prime}(h(y)) \psi(y)\right|}{|x-y|^{1+\alpha \hat{q}}} \mathrm{~d} x \mathrm{~d} y .
\end{aligned}
$$


We now give an upper bound for the term $J_{2}$ : notice first that, thanks to the fact that $\alpha \hat{q}>1$, the space $\mathcal{W}_{0}^{\alpha, \hat{q}}$ is continuously imbedded in $\mathcal{C}_{0}([0,1])$. Thus

$$
\|\psi\|_{\infty} \leq c\|\psi\|_{\mathcal{W}_{0}^{\alpha, \hat{q}}} \quad \text { and } \quad\|w\|_{\infty} \leq c\|w\|_{\mathcal{W}_{0}^{\alpha, \hat{q}} .}
$$

Now, plugging (44) into the definition of $J_{2}$, it is readily checked that

$$
J_{2} \leq c\left\|\sigma^{(2)}\right\|_{\infty}\|h\|_{\mathcal{W}_{0}^{\alpha, \hat{q}}}\|w\|_{\mathcal{W}_{0}^{\alpha, \hat{q}}}\|\psi\|_{\mathcal{W}_{0}^{\alpha, \hat{q}}} .
$$

The terms $J_{1}, J_{3}$ and $J_{4}$ can now be handled along the same lines, finishing the proof of (43). Thus, Hypothesis 1 is satisfied in our example. In order to apply Theorem पin our situation, it remains to verify (22). This is left to the reader, since it can be done with the same kind of arguments as above.

Remark 3. Notice that for $H>1 / 2$, one is still allowed to choose $q_{n}=1$, that is a white noise in time.

Remark 4. It is also easy to construct an equation of the form (33) for which the coefficient $B$ is non trivial, but still globally Lipschitz, leading thus to an infinite explosion time: just choose a test function $\varphi:[0,1] \in \mathbb{R}$, another function $\sigma \in C_{b}^{2}(\mathbb{R})$, and set

$$
B(w)=\int_{0}^{1} \sigma(w(x)) \varphi(x) \mathrm{d} x,
$$

which defines a map from $\mathcal{W}_{0}^{\delta, p}$ to $\mathcal{L}\left(\mathcal{W}_{0}^{-\alpha, \hat{p}} ; \mathcal{W}_{0}^{-\alpha, p}\right)$ satisfying the desired global Lipschitz conditions. The proof of this claim is left to the reader.

\section{References}

[Ada75] R. A. Adams. Sobolev spaces. Academic Press, 1975.

[DPZ92] G. Da Prato and J. Zabczyk. Stochastic equations in infinite dimensions, volume 44 of Encyclopedia of Mathematics and its Applications. Cambridge University Press, Cambridge, 1992.

[EN00] K.-J. Engel and R. Nagel. One-parameter semigroups for linear evolution equations, volume 194 of Graduate Texts in Mathematics. Springer-Verlag, New York, 2000.

[Fat99] H. O. Fattorini. Infinite-dimensional optimization and control theory, volume 62 of Encyclopedia of Mathematics and its Applications. Cambridge University Press, Cambridge, 1999. 
[Gub04] M. Gubinelli. Controlling rough paths. Jour. Funct. Anal., to appear, 2004.

[Lej03] A. Lejay. An introduction to rough paths. In Séminaire de Probabilités XXXVII, volume 1832 of Lecture Notes in Mathematics, pages 1-59. Springer-Verlag Heidelberg, 2003.

[LLQ02] M. Ledoux, T. Lyons, and Z. Qian. Lévy area of Wiener processes in Banach spaces. Ann. Probab., 30(2):546-578, 2002.

[LQ02] T. Lyons and Z. Qian. System control and rough paths. Oxford University Press, 2002.

[Lyo98] T. Lyons. Differential equations driven by rough signals. Rev. Mat. Iberoamericana, 14(2):215-310, 1998.

[MN03] B. Maslowski and D. Nualart. Evolution equations driven by a fractional Brownian motion. J. Funct. Anal., 202(1):277-305, 2003.

[Paz83] A. Pazy. Semigroups of linear operators and applications to partial differential equations, volume 44 of Applied Mathematical Sciences. Springer-Verlag, New York, 1983.

[TTV03] S. Tindel, C. A. Tudor, and F. Viens. Stochastic evolution equations with fractional Brownian motion. Probab. Theory Related Fields, 127(2):186-204, 2003.

[Wal86] J. B. Walsh. An introduction to stochastic partial differential equations. In École d'été de probabilités de Saint-Flour, XIV-1984, volume 1180 of Lecture Notes in Math., pages 265-439. Springer, Berlin, 1986.

[You36] L. C. Young. An inequality of hölder type, connected with stieljes integration. Acta Math., 67:251-282, 1936.

[Zäh98] M. Zähle. Integration with respect to fractal functions and stochastic calculus. I. Probab. Theory Related Fields, 111(3):333-374, 1998. 